J. Clin. Chem. Clin. Biochem.

Vol. 16, 1978, pp. 225-230

\title{
Mikrobestimmung von Glucose mit Glucosedehydrogenase bei bestimmungsunabhängiger Proben- vorbereitung im Routinelaboratorium
}

Von W. Stein, I. Mildner und R. Maulbetsch

Aus der Medizinischen Klinik, Abteilung für Innere Medizin IV (Direktor Professor Dr. M. Eggstein) Tübingen

(Eingegangen am 4. Juli/6. Oktober 1977)

\section{Herrn Professor Dr. med. Dr. h. c. Gotthard Schettler zum 60. Geburtstag}

Zusammenfassung: Eine Mikromethode zur Bestimmung der Glucose im Blut mittels Glucosedehydrogenase im Routinelaboratorium wird für den Autoanalyzer II und als manuelle Methode beschrieben. Die Probennahme von $20 \mu \mathrm{l} \mathrm{Kapillarblut} \mathrm{und} \mathrm{Enteiweißung} \mathrm{mit} \mathrm{Uranylacetat} \mathrm{vereinfacht} \mathrm{den} \mathrm{Analysengang} \mathrm{am} \mathrm{Autoanalyzer} \mathrm{II} \mathrm{und} \mathrm{er-}$ möglicht auch die manuelle Verarbeitung derselben Probe. Präzision und Richtigkeit sind der Bestimmung mit der Hexokinase-Glucose-6-phosphatdehydrogenase-Methode vergleichbar. Elf-monatige Erfahrung zeigt Praktikabilität und ökonomische Handhabung der Methode.

\section{Microdetermination of glucose using glucose dehydrogenase, with independent sample preparation in the routine laboratory}

Summary: A micromethod for the determination of glucose in $20 \mu \mathrm{l}$ of capillary blood using glucose dehydrogenase is described. After deproteinisation with uranyl acetate, the samples are analysed by an Autoanalyzer II method or by a manual procedure. Precision and accuracy are well correlated with the hexokinase-glucose-6-phosphate dehydrogenase method. Eleven months experience have shown the practicability and economic advantages of this method.

\section{Einleitung}

Die Glucoseoxidase-Perid-Methode erbrachte in unserem Laboratorium einen Variationskoeffizienten von knapp $5 \%$ für die Glucosebestimmung in Kapillarblut. Den Ansprüchen an einfache und schnelle Handhabung wurde sie nicht mehr gerecht.

Eine Nachfolgemethode muß folgenden Forderungen entsprechen:

1. einfach durchführbare, gegen Störungen wenig empfindliche chemische Reaktionen mit geringem Enzymverbrauch,

2. geringe Probenmenge und Probennahme auch durch nichtärżtliches Personal,

3. uniforme Probenvorbereitung und -entnahme, d. h. manuelle bzw. seriell-mechanisierte Bestimmungen sollten keine unterschiedlichen Probennahmen und Probenvorbereitungen verlangen,

4. positive Probenidentifizierung.

Mit der Glucosedehydrogenase $\left.{ }^{1}\right)$-Methode $(1,2,3,4)$ steht eine Glucosebestimmung zur Verfügung, die diese

1) B-D-Glucose: NAD-Oxidoreductase EC 1.1.1.47. Mutarotase: Aldose-1-epimerase EC 5.1.3.3.
Voraussetzungen erfüllen und die als Fortschritt zu bisherigen Verfahren empfohlen werden kann.

Die kapillare Mikro-Probennahme (5) wird seit Jahrzehnten bei uns bevorzugt; sie berücksichtigt die klinisch praktischen Belange (Kinder, engmaschiges Profil) und erlaubt auch die Selbstabnahme durch den Patienten.

Eine Enteiweißung der Blutproben vereinfacht den Analysengang der Methode und kann für Autoanalyzer und manuelle Bestimmung in gleicher Weise erfolgen. Außerdem läßt sich der Überstand nach Enteiweißung auch zur Glucosebestimmung am Automatischen Clinischen Analysator ACA (Dupont) verwerten (6).

Das direkte Einsetzen des Abnahmegefäßes in den Probenteller des Autoanalyzers II ermöglicht eine positive Probenidentifizierung, wie sie bei größeren Meßserien und Rechnereinsatz wünșchenswert ist (7).

\section{Verbrauchsmaterial und Geräte}

\section{Materialien}

Lanzetten (Firma ASID, München).

Heparinisierte Glaskapillaren $20 \mu \mathrm{l}$ (Firma Biotechnik, Hamburg). Mikrogefäße (Firma Eppendorf, Hamburg).

Spezialpapier für den Schreiber (Firma Blumberg, Ratingen). 


\section{Reagenzien}

Arbeitslösungen für die Glucosedehydrogenase-Methode

1. Enteiwcißungsmittel

1.1 Uranylacetat $(1,9 \mathrm{mmol} / \mathrm{l})$ in Natriumchloridlösung $(154 \mathrm{mmol} / \mathrm{l}): 4 \mathrm{~g}$ Uranylacetat $\times 2 \mathrm{H}_{2} \mathrm{O}$ werden in 5 Liter physiologischer Natriumchloridlösung aufgelöst.

1.2 Perchlorsäure/Perchloratlösung (Merck Nr. 9431) für die Bcstimmung von Lösungen mit geringem Eiweißgehalt.

2. Reaktionslösung Aus Merck System Reagenzien Nr. 14051 und 14055 werden in 11 Puffer 2 Flaschen Enzymgemisch $(5,2 \mathrm{kU} / \mathrm{l}$ Glucosedehydrogenase und $0,11 \mathrm{kU} / 1$ Mutarotase), 2 Flaschen NAD $(1,1 \mathrm{mmol} / \mathrm{l})$ sowie $1 \mathrm{ml}$ Triton X 100 gelöst.

3. Als Glucosestandards werden Lösungen der Firmen Merck (Nr. 9423) und Boehringer (Preciset Glucose) eingesetzt und $2+50$ mit bidestilliertem Wasser oder Lösung 1.2 verdünnt.

4. Spüllösung für den Probennehmer 11 bidestilliertes Wasser wird mit $1 \mathrm{ml}$ Triton $X 100$ versetzt.

5. Reinigungslösung

Zur täglichen Reinigung des Leitungssystems des Gerätes wird alkalische Hypochloritlösung $(40 \mathrm{~g} / \mathrm{l})$ verwendet. (Verdünnung der Natronbleichlauge $125 \mathrm{~g} / \mathrm{kg}$ der Firma Nettenheimer u. Simon-Collischon, Frankfurt).

\section{Reagenzien für Vergleichsmessungen}

Hexokinase-Glucose-6-phosphatdehydrogenase: Reagenzien von Boehringer, Mannheim (Nr. 15931).

Glucoseoxidase-Perid-Reagenzien von Boehringer, Mannheim (Nr. 124044).

\section{Geräte}

Die mechanisierten Bestimmungen werden auf einem EinkanalAutoanalyzer II der Firma Technicon, bestehend aus Pumpe II, Kolorimeter mit $15 \mathrm{~mm}$ Durchflußküvette und Filter $340 \mathrm{~nm}$ durchgeführt. Der Probenteller von IBM (Sampler Recorder 1084) gewährleistet die Probenidentifizierung und Steuerung des Auswerteprogramms über einen eingebauten Kurzlochkartenleser (7). Der Einkanalschreiber wird mit Schreiberpapier mit Einteilungen von $2,5 \mathrm{mg} / \mathrm{dl}$ und einem Bereich von 0-500 mg/dl bzw. 0-27,78 mmol/l Glucose betrieben.

Das Fließschema des Analyzers zeigt Abbildung 1. Vom zentrifugierten, enteiweißten Überstand der Proben wird 50 Sekunden lang abgesaugt, mit Lösung 2 versetzt und mit Luft segmentiert. Die Reaktion läuft während des Durchflusses durch die Mischund Verweilspiralen innerhalb 13 Minuten ab. Diese Zeit ist ausreichend, um selbst bei Proben mit einer Glucosekonzentration von $25 \mathrm{mmol} / \mathrm{l}$ bei längerem Absaugen ein stabiles Plateau zu erhalten, sodaß kleinere Veränderungen in der Reaktionsgeschwindigkeitskonstanten nicht in das Ergebnis eingehen können. Anschließend wird das gebildete NADH bei $340 \mathrm{~nm}$ nach Abtrennung der Luft in der Durchflußzelle gemessen.

Einzelbestimmungen werden am Photometer Eppendorf (M 1101) manuell durchgeführt.

\section{Durchführung und Ergebnisse der Analysen}

\section{Kapillartechnik}

Die Probennahme - Kapillarblut - erfolgt mit einer $20 \mu \mathrm{l}$ Glaskapillare, die luftblasenfrei ohne äußere Benetzung gefullt wird. Die gesamte Kapillare wird sofort in ein Mikrogefäß mit $500 \mu \mathrm{l}$ Enteiweißungslösung gegeben, und durch gründliches Schütteln des verschlossenen Gefäßes wird die Probe aus der Kapillare in die Enteiweißungslösung überführt. Eiweißarme und eiweißfreie Lösungen werden mit Lösung 1.2 verdünnt. Der Eiweißniederschlag wird abzentrifugiert ( 1 Minute, $7500 \mathrm{~g}$ ). Die Probe ist damit für die mechanisierte Analyse oder für die manuelle Bestimmung vorbereitet.

Zur Abschätzung eines möglichen Fehlers, der durch die Kapillartechnik bei der Probennahme verursacht wird, wurde ein Glucosestandard aus dem kritischen Bereich von $2,8 \mathrm{mmol} / \mathrm{l}$ Glucose eingesetzt.

Eine Zehnfachanalyse in Kapillartechnik und der direkte Einsatz des $2+50$ vorverdünnten Standards ergab die gleichen Werte (Tab. 1).

\section{Analysen mit dem Autoanalyzer II}

\section{Durchführung der Analysen}

Die Proben werden mit einer Frequenz von $60 / \mathrm{h}$ aus den Mikrogefäßen angesaugt und bestimmt. Das Saug-/Waschverhältnis beträgt $50: 10$.

Die Kalibrierung des Gerätes erfolgt an zwei Punkten. Zwei Proben mit destilliertem Wasser und zwei Glucosestandards 300 $(300 \mathrm{mg} / \mathrm{dl}$ entsprechend $16,67 \mathrm{mmol} / \mathrm{l}$ ) werden zu Beginn jeder Serie mitgeführt, um Photometer und Schreiber auf die Sollwerte 0 und 300 einstellen zu können.

Die Präzision wird durch ein Testserum in jeder Serie geprüft (Monitrol II Lot Nr. XPT 62, Merz und Dade).

Die Richtigkeit wird täglich mit Precinorm U (606, Boehringer, Mannheim) kontrolliert.

Jede Serie setzt sich aus zwei Leerwerten, zwei Glucosestandards, bis zu 20 Proben und einem Testserum zusammen. Ein konstanter Durchfluß im Autoanalyzer mit $817 \pm 20$ Sekunden Durchlaufzeit, die für die direkte Probenidentifizierung und Auswertung Voraussetzung ist, wird sicher gewährleistet. ( $\mathrm{N}=40$; $\bar{x}=813 \mathrm{~s}$; Standardabweichung: $4 \mathrm{~s}$; Variationskoeffizient: $0,4 \%)$.

Die Auswertung erfolgt on-line durch das EDV-System der Klinik (7), die Serien werden durch eine Laborschreibmaschine ausgedruckt.

\section{Linearität und Richtigkeit}

Die Linearität wurde durch Analysen wäßriger Glucosestandardlösungen überprüft. Abbildung 2 zeigt, daß bis

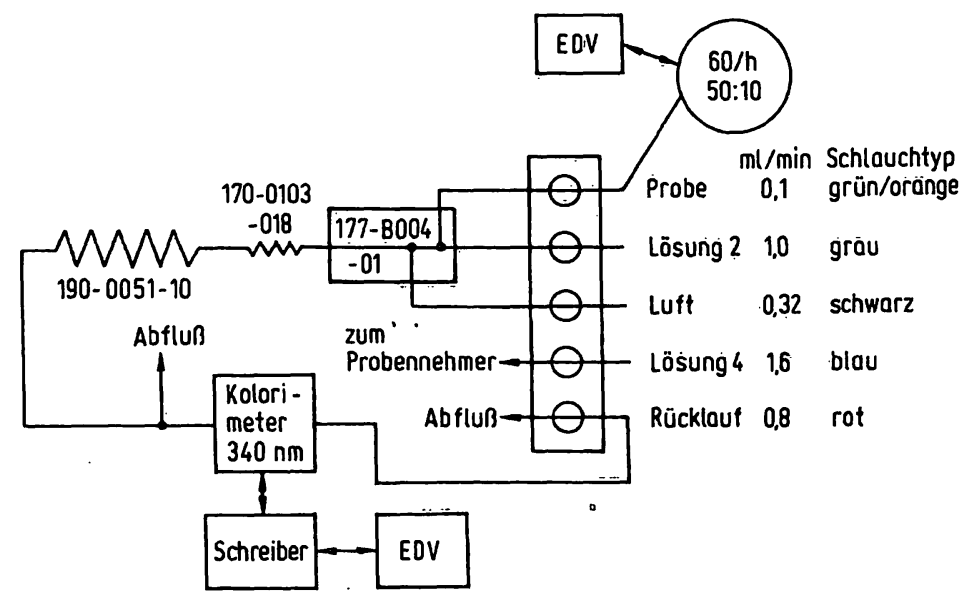

Abb. 1. Fließschema zur Glucose-Bestimmung mit Glucosedehydrogenase auf dem Autoanalyzer II. 


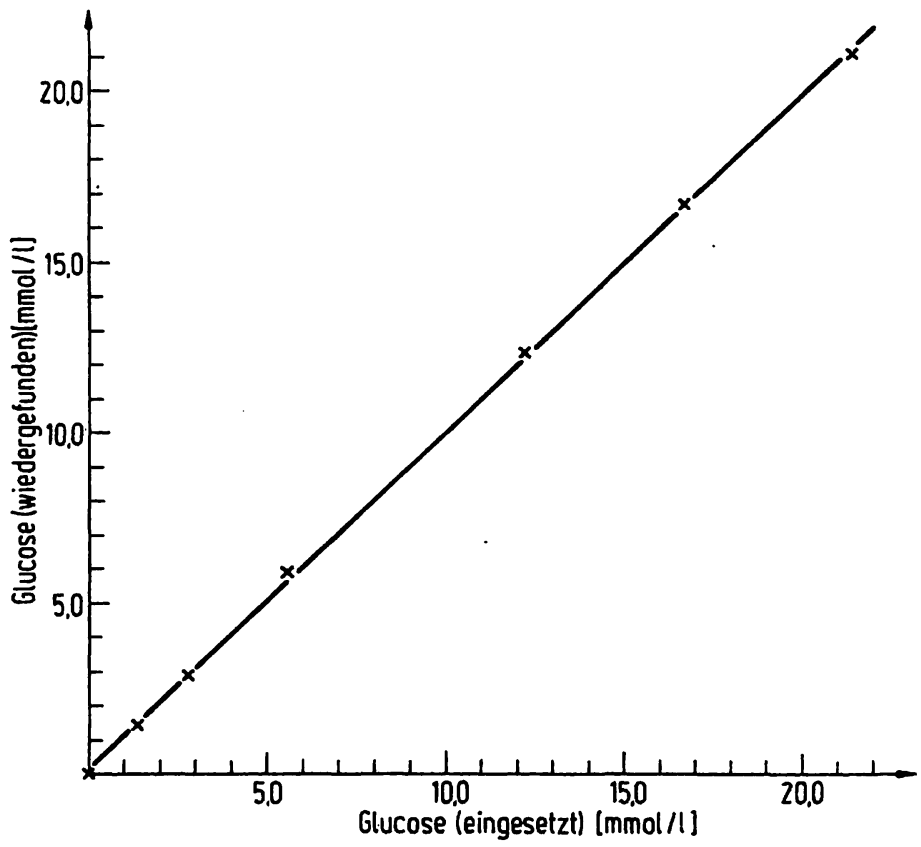

Abb. 2. Glucose-Bestimmung mit Glucosedehydrogenase auf dem Autoanalyzer Iİ. Prüfung der Linearität und Richtigkeit mit wäßrigen Standardlösungen.

Tab. 1. Einfluß der Probennahme mit einer $20 \mu \mathrm{l}-\mathrm{Kapillare}$ auf Präzision und Richtigkeit.

\begin{tabular}{lllll}
\hline & $\begin{array}{l}\text { Glucose } \\
\bar{x} \\
(\mathrm{mmol} / \mathrm{l})\end{array}$ & $\begin{array}{l}\text { s } \\
(\mathrm{mmol} / \mathrm{l})\end{array}$ & $\begin{array}{l}\text { VK } \\
(\%)\end{array}$ & $\mathrm{N}$ \\
\hline Kapillartechnik & 2,86 & 0,03 & 1,0 & 10 \\
Vorverdünnung 2+50 & 2,85 & 0,03 & 1,0 & 10 \\
\hline
\end{tabular}

zu Konzentrationen von $22 \mathrm{mmol} / 1$ Glucose die theoretische Menge wiedergefunden wird.

Die Richtigkeit für Serumanalysen wurde an Hand von Kontrollseren festgestellt. Die erhaltenen Werte sind in Tabelle 2 aufgeführt. Unsere Bestimmungen weichen bei den Seren nur um maximal $2,5 \%$ von den angegebenen Mittelwerten ab.

\section{Präzision}

Die Untersuchungen zur Präzision wurden in einem Zeitraum von sieben Monaten von verschiedenen Assistentinnen während des Routinebetriebs durchgeführt.

Als Gerätepräzision wird hier die für eingewogene, $2+50$ vorverdünnte Standards ermittelte Präzision in der Serie bezeichnet. Im Bereich von $22,51 \mathrm{mmol} / \mathrm{l}$ beträgt die Standardabweichung 0,13 mmol/1 $(\mathrm{N}=20)$. Bei einer Konzentration von 1,6 mmol/1 Glucose nimmt die Standardabweichung den Wert $0,18 \mathrm{mmol} / 1$ an $(\mathrm{N}=10)$.

Die Meßwerte für die Präzision in der Serie schließen den Probennahmefehler mittels Kapillartechnik ein. Für drei Seren verschiedener Glucosekonzentrationen sind die Ergebnisse in Tabelle 3 zusammengestellt.

Für die Langzeitpräzision gibt Tabelle 4 die Ergebnisse mit fünf Testseren und einem Glucosestandard wieder. Die Langzeitmessungen $(8,9)$ an Monitrol Il Lot XPT 62 wurden mit der Glucosedehydrogenase-Methode begonnen, nachdem schon etwa 300 Meßwerte mit der Glucoseoxidase-Perid-Methode am Autoanalyzer vorlagen. Diese Methodenumstellung erbrachte eine Verbesserung des Variationskoeffizienten von 4,1 auf $2,3 \%$.

Tab. 2. Richtigkeitskontrolle mit käuflichen Seren und Standards.

\begin{tabular}{|c|c|c|c|}
\hline & $\begin{array}{l}\text { Glucose } \\
\text { Sollwert } \\
(\mathrm{mmol} / \mathrm{l})\end{array}$ & $\begin{array}{l}\text { Istwert } \\
\bar{x}(\mathrm{mmol} / \mathrm{l})\end{array}$ & Bemerkung \\
\hline $\begin{array}{l}\text { Monitrol II } \\
(46 \mathrm{~A})\end{array}$ & $\begin{array}{l}11,8 \\
\text { (Glucosedehydro- } \\
\text { genase) }\end{array}$ & 11,9 & Mittelwert aus sechsfacher Bestimmung an 2 Tagen \\
\hline $\begin{array}{l}\text { Precinorm U } \\
(606)\end{array}$ & $\begin{array}{l}6,22 \\
\text { (Glucosedehydro- } \\
\text { genase) }\end{array}$ & 6,37 & Mittelwert aus zehnfacher Bestimmung \\
\hline $\begin{array}{l}\text { Fluinorm N } \\
\text { (127 D) }\end{array}$ & $\begin{array}{l}5,45 \\
\text { (Hexokinase) }\end{array}$ & 5,33 & $\begin{array}{l}\text { Mittelwert aus Einzelbestimmungen an } 55 \text { aufeinanderfolgenden Werk- } \\
\text { tagen }\end{array}$ \\
\hline $\begin{array}{l}\text { Monitrol I } \\
(137 \mathrm{~A}, \mathrm{~B})\end{array}$ & $\begin{array}{l}4,33 \\
\text { (Glucosedehydro- } \\
\text { genase) }\end{array}$ & 4,45 & Doppelbestimmung \\
\hline Standard 50 & $\begin{array}{l}2,78 \\
\text { (Einwaage) }\end{array}$ & 2,86 & Mittelwert aus zwanzigfacher Bestimmung \\
\hline Standard 25 & $\begin{array}{l}1,4 \\
\text { (Einwaage) }\end{array}$ & 1,6 & Mittelwert aus zehnfacher Bestimmung \\
\hline
\end{tabular}


Tab. 3. Präzision in der Seric

\begin{tabular}{llll}
\hline Glucose & & & \\
$\bar{x}(\mathrm{mmol} / \mathrm{l})$ & $\mathrm{s}(\mathrm{mmol} / \mathrm{l})$ & $\mathrm{VK}(\%)$ & $\mathrm{N}$ \\
\hline 12,22 & 0,15 & 1,2 & 17 \\
6,50 & 0,03 & 0,4 & 20 \\
2,91 & 0,07 & 2,4 & 15 \\
\hline
\end{tabular}

Die Verschleppung im Analysengerät läßt sich durch eine Testserie aus primären Standards mit einer Konzentration von $16,67 \mathrm{mmol} / 1$ und $2,86 \mathrm{mmol} / 1$ nach $\mathrm{Hjelm}$ (10) demonstrieren. Sie ist gering; der Interaktionskoeffizient beträgt $q=0,006$.

\section{Störungen}

Hämolyse: Hämoglobinkonzentrationen im Plasma bis $20 \mathrm{~g} / \mathrm{l}$ sind ohne Einfluß auf das Ergebnis. Stärkere Hämolyse führt infolge Absorption des Hämoglobins zu falsch hohen Werten.

Bilirubin: Bilirubinkonzentrationen bis $260 \mu \mathrm{mol} / 1$ Serum sind ohne Einfluß. Bei Bilirubinwerten von 700 $\mu \mathrm{mol} / \mathrm{l}$ werden die Glucosekonzentrationen um etwa $0,5 \mathrm{mmol} / 1$ erhöht ermittelt.

Lipämie: Triglyceridkonzentrationen von $35 \mathrm{mmol} / \mathrm{l}$ Serum waren ohne Einfluß.

Eiweiß: Im Bereich von $40 \mathrm{~g} / 1$ bis $120 \mathrm{~g} / 1$ Eiweiß im Serum sind keine Einflüsse nachzuweisen. Eiweißkonzentrationen unter $30 \mathrm{~g} / \mathrm{l}$ führen bei Enteiweißung mit Uranylacetat zu Veränderungen im Schreiberbild in Form von schmalen, auf die Peaks aufgesetzten Zacken.

\section{Methodenvergleich}

Die guten Übereinstimmungen der beschriebenen Methode mit anderen Mikromethoden wurde durch Vergleichsmessungen an identischen Blutproben bzw. Liquor bewiesen.

Abbildung 3 zeigt den Vergleich mit der manuellen Hexokinase-Glucose-6-phosphatdehydrogenase-Methode (11) an 172 Wertepaaren.

Im Vergleich mit der Glucoseoxidase-Perid-Methode (12) errechnet sich die Regressionsgerade $y=b x+a z u$ $y=0,929 x+0,483$ und der Korrelationskoeffizient $z u$

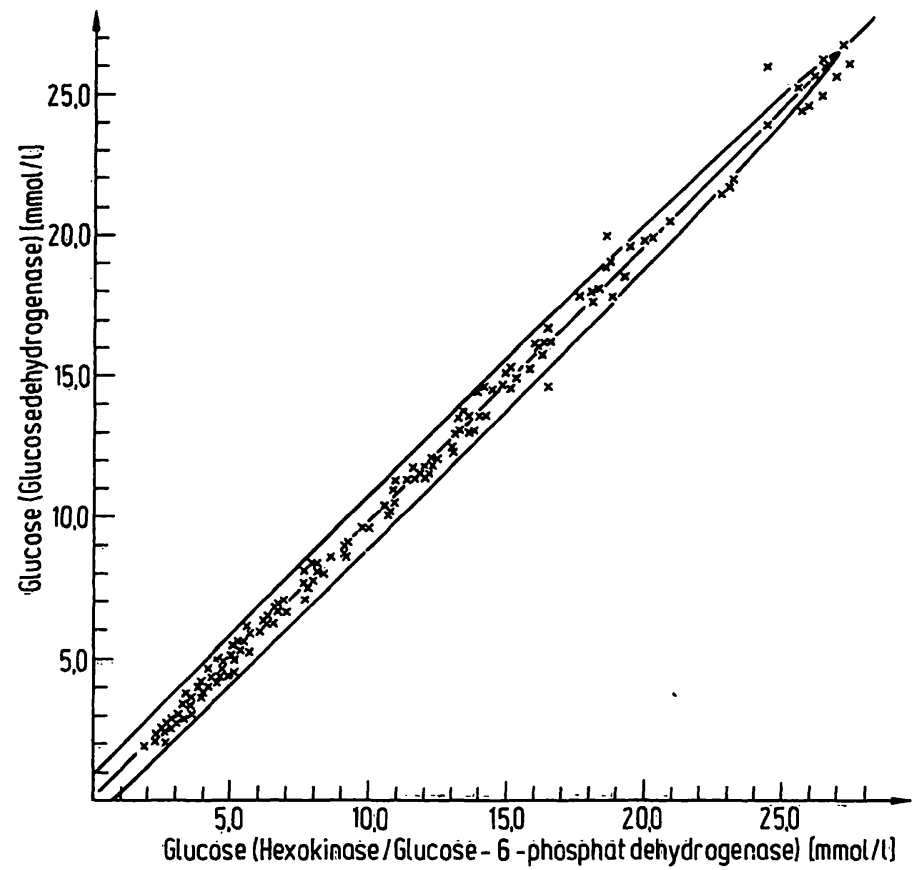

Abb. 3. Glucosebestimmung in Vollblut

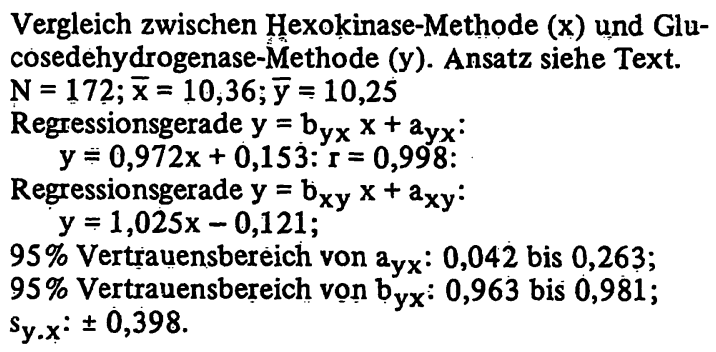

$\mathrm{r}=0,994 . \mathrm{N}=113$ Wertepaare; $\overline{\mathrm{x}}=7,29 ; \overline{\mathrm{y}}=7,25 ; 95 \%$ Vertrauensbereich von a $=0,299$ bis 0,$666 ; 95 \%$ Vertrauensbereich von $b=0,907$ bis 0,$949 ; s_{\mathbf{y}} \cdot \mathbf{x}=0,494$.

Vergleiche der mechanisierten (y) mit der manuellen (x) Glucosedehydrogenase-Methode ergeben bei $N=136$ Wertepaaren folgendes Ergebnis: $y=0,982 x+0,136$; $\bar{x}=8,06 ; \bar{y}=8,05 ; r=0,999 ; 95 \%$ Vertrauensbereich von a $=0,052$ bis 0,$220 ; 95 \%$ Vertrauensbereich von $\mathrm{b}=0,974$ bis 0,$990 ; \mathrm{s}_{\mathbf{y}} \cdot \mathrm{x}=0,272$.

Manuelle Bestimmungen mit Glucosedehydro: genase

Die Proben werden hierbei einzeln mit dem gleichen Reaktionsgemisch (Lösung 2) wie bei der mechanisierten Analyse bestimmt. $0,1 \mathrm{ml}$ Überstand nach Enteiweißung werden mit $1,0 \mathrm{ml}$ Lösung 2 bei Raumtemperatur 10-15 Minuten inkubiert.

Tab. 4. Langzeitpräzision

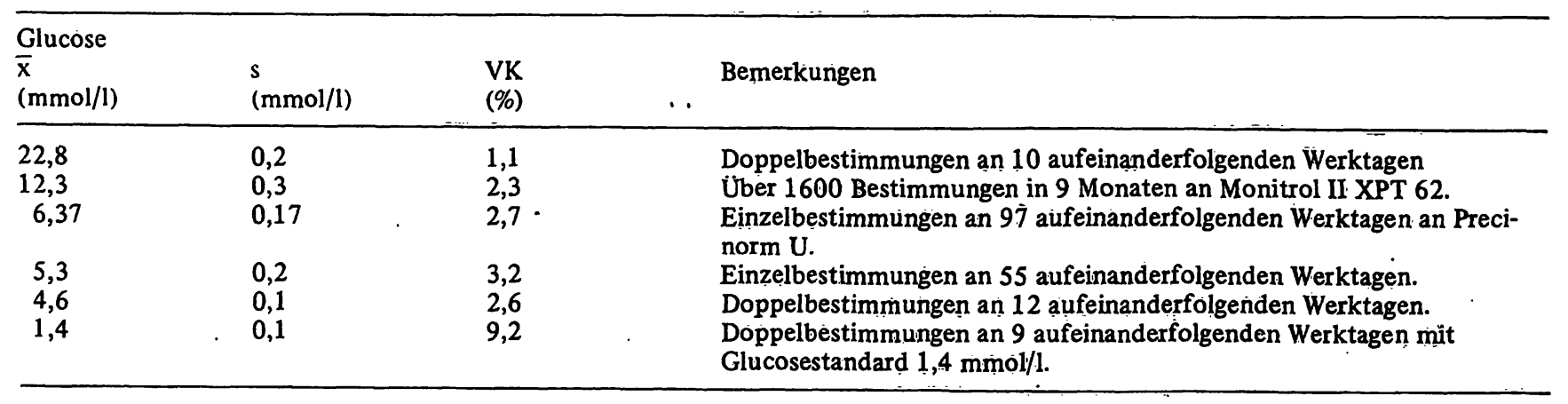


Tab. 5. Ergebnisse der manuellen Bestimmung mit Glucosedehydrogenase.

\begin{tabular}{llllll}
\hline $\begin{array}{l}\text { Glucose } \\
\begin{array}{l}\text { Sollwert } \\
(\mathrm{mmol} / \mathrm{l})\end{array}\end{array}$ & $\begin{array}{l}\text { Istwert } \\
\overline{\mathbf{x}}(\mathrm{mmol} / \mathrm{l})\end{array}$ & $\mathrm{N}$ & $\begin{array}{l}\text { s } \\
(\mathrm{mmol} / \mathrm{l})\end{array}$ & $\begin{array}{l}\text { VK } \\
(\%)\end{array}$ & Bemerkungen \\
\hline 1,39 & 1,4 & 20 & 0,1 & 6,7 & wäßr. Glucosestandard \\
2,78 & 2,9 & 7 & 0,1 & 3,1 & wäßr. Glucosestandard \\
5,56 & 5,7 & 4 & 0,1 & 2,4 & wäßr. Glucosestandard \\
16,67 & 16,3 & 6 & 0,3 & 1,7 & wäßr. Glucosestandard \\
27,78 & 27,6 & 6 & 0,4 & 1,4 & wäßr. Glucosestandard \\
55,56 & 54,6 & 18 & 0,9 & 1,7 & Einzelbestimmungen während \\
& 12,2 & 99 & 0,2 & & 25 Tagen mit Monitrol II \\
& & & & & XPT 62. \\
\hline
\end{tabular}

Anschließend wird die Absorbanz $\left(A_{334}\right)$ der Probe gegen einen Reagenzienleerwert aus $1,0 \mathrm{ml}$ Lösung 2 und $0,1 \mathrm{ml}$ Wasser bei $334 \mathrm{~nm}$ und einem Zentimeter Schichtdicke gemessen. Pro Serie genügt ein Leerwert.

Die Berechnung der Glucosekonzentration erfolgt nach:

$$
\begin{aligned}
& c=A_{334} \times 46(\mathrm{mmol} / \mathrm{l}) \text { oder } \\
& c=A_{334} \times 828(\mathrm{mg} / \mathrm{dl}) .
\end{aligned}
$$

Für die manuelle Methode werden mit der Reaktionslösung 2 die gleichen Ergebnisse wie in der Literatur beschrieben (1) erzielt, das zugesetzte Triton X 100 ist ohne Einfluß. Die Enzymkonzentration im Ansatz und die Reaktionszeit bei Raumtemperatur reichen für die Bestimmung bis zu Glucosekonzentrationen von $55 \mathrm{mmol} / \mathrm{l}$ ohne Vorverdünnung aus. Auch ein Überschreiten der Reaktionszeit bei Raumtemperatur um 5-10 Minuten führt nur zu einem vernachlässigbaren Absinken der Absorbanz bei $334 \mathrm{~nm}$. Unsere Ergebnisse für Richtigkeit, Linearität und Präzision sind in Tabelle 5 zusammengefaßt. Der Methodenvergleich $z$ wischen manueller GlucosedehydrogenaseMethode (y) und manueller Hexokinase-Glucose-6-phosphatdehydrogenase-Methode $(x)$ brachte folgende Ergebnisse:

$\mathrm{N}=46 ; \overline{\mathrm{x}}=11,46 ; \overline{\mathrm{y}}=11,23 ; \mathrm{y}=0,997 \mathrm{x}-0,193 ; \mathrm{r}=0,999$; $95 \%$ Vertrauensbereich von a: $-0,044$ bis $-0,342 ; 95 \%$ Vertrauensbereich von $b: 0,989$ bis 1,$006 ; s_{y} \cdot x= \pm 0,057$.

\section{Diskussion}

Das beschriebene System zur Glucosebestimmung wird den Anforderungen hinsichtlich Praktikabilität, Richtigkeit und Präzision gerecht (Tab. 2,4; Abb. 2,3).

Die einheitliche Probennahme und -vorbereitung, die unabhängig von einer sich anschließenden Routine- oder Notfalluntersuchung ist, hat sich bewährt; wir erhalten im Autoanalyzer oder mit der manuellen Methode direkt vergleichbare Resultate bei einem Kapillarblutbedarf von $20 \mu l$.

Unterschiede in der Glucosekonzentration zwischen venösem Blut und Kapillarblut (13) sowie Serum und Vollblut verlangen eine einheitliche Probennahme. Unsere Ergebnisse zeigen, daß die kapillare Abnahme keine meßbare Verschlechterung der Präzision bedingt. Dàmit bildet diese Technik für Patient, Klinik und Labor auch fernerhin die Methode der Wahl.
Die Glucosedehydrogenase-Methode wird sowohl am Autoanalyzer II als auch manuell mit der gleichen Reaktionslösung durchgeführt. Sie ist bei Raumtemperatur mindestens vier Wochen stabil, damit länger haltbar und auch preiswerter als andere enzymatische Verfahren. Der Analysenbereich reicht beim Autoanalyzer II bis 25 mmol/l, bei der manuellen Bestimmung besteht Linearität bis über $50 \mathrm{mmol} / \mathrm{l}$ Glucose. Proben mit geringem Glucosegehalt können bis in den Bereich 1,25 mmol/1 Glucose noch präzis und richtig bestimmt werden. Uranylacetat zur Enteiweißung ist gegenüber Perchlorsäure weniger aggressiv und bildet einen besser abzentrifugierbaren Niederschlag. Durch die Enteiweißung wird ein Hämolysierschritt und/oder Dialysierschritt erspart, mit dem Vorteil größerer Betriebssicherheit und sofortiger Inaktivierung der Glykolyse. Der Volumenverdrängungseffekt durch die Enteiweißung liegt wegen der Verdünnung von 1:26 mit etwa $0,6 \%$ in der gleichen Größenordnung wie bei üblichen Serumanalysen mit Enteiweißung und wird allgemein in Kauf genommen (2).

Der zur mechanisierten Analyse verwendete Autoanalyzer II ist im Aufbau unkompliziert, leicht zu bedienen und problemlos in der Wartung. Dialysatoren und Thermostaten werden nicht benötigt. Die einzigen Verschleißteile sind 5 Pumpenschläuche, deren monatliche Erneuerung für einen sicheren Betrieb ausreicht.

Dominierend für die Verbrauchskosten einer Glucosebestimmung sind die benötigten Enzymmengen. Das beschriebene Verfahren kommt mit einer Menge von 5,2 U Glucosedehydrogenase pro Probe aus, während entsprechende Verfahren am Autoanalyzer die doppelte Menge: 10 (4) bzw. 12,6 (1) U Glucosedehydrogenase pro Probe umsetzen.

Unser System zur Glucosebestimmung mit Glucosedehydrogenase ist seit etwa einem Jahr bei 200-250 Glucosebestimmungen pro Werktag und bei 50-100 Werten an Sonn- oder Feiertagen im Einsatz. 


\section{Literatur}

1. Banauch, D., Brümmer, W., Ebeling, W., Metz, H., Rindfrey, H., Lang, H., Leybold, K. \& Rick, W. (1975) Z. Klin. Chem. Klin. Biochem. 13,101-107.

2. Gerbig, K. (1976), Medizin. Laboratorium 29, 1-12.

3. Dolhofer, R., Weiss, L. \& Wieland, O. H. (1976), J. Clin. Chem. Clin. Biochem. 14, 415-417.

4. Scholer, A. \& Pianezzi, A. J. (1976), J. Clin. Chem. Clin. Biochem. 14, 189-195.

5. Richterich, R., Klinische Chemie (1965), Akademische Verlagsgesellschaft Frankfurt, p. 190.

6. Stein, W., in Vorbereitung.

7. Bock, H. E. \& Eggstein, M. (Hrsg.) (1970), Diagnostik Informationssystem, Springer Verlag Berlin, Heidelberg, New York.

8. Schmülling, R., Gräser, W. \& Eggstein, M. (1975), Organisation des laboratoires - biologie prospective III $^{\mathrm{e}}$ collegue de

Pont-a-Mousson. L'expansion scientifique française, éditeur p. $147-156$.

9. Schmülling, R., Gräser, W. \& Eggstein, M. (1975), in: Methoden der Informatik in der Medizin (Reichertz, P. L. \& Holthoff, G. eds.); Springer Verlag Berlin, Heidelberg, New York p. 159-169.

10. Hjelm, M., (1968), Z. Anal. Chem. 243, 781-790.

11. Bergmeyer, H. U., Bernt, E., Schmidt, F. \& Stork, H. (1974), in: Methoden der enzymatischen Analyse (Bergmeyer, H. U. ed.) Vol. II, p. 1241-1246. Verlag Chemie Weinheim.

12. Werner, W., Rey, H. G. \& Wielinger, H. (1970), Z. Anal. Chem. 252, 224=228.

13. Kaplan, S. A., Yuceoglu, A. M. \& Straus, J. (1959), Pediatrics 24, 270-276.

Dr. rer. nat. Wolfgang Stein Medizinische Klinik, Abt. IV Otfried-Müller-Str. 10 7.400 Tübingen 